\title{
Studies Investigating Fetal Alcohol Spectrum Disorders in the Criminal Justice System: A Systematic PRISMA Review
}

\author{
Clare S. Allely ${ }^{*}$ and Piersanti Gebbia ${ }^{2}$ \\ ${ }^{1}$ Centre for Health Sciences Research, University of Salford, Salford, England M6 6PU; and affiliate member of the Gillberg Neuropsychiatry Centre, \\ Sahlgrenska Academy, University of Gothenburg, Gothenburg, Sweden \\ ${ }^{2}$ Psychology Graduate and Alumni of the University of Salford, Salford, England
}

Received: January 18, 2016; Accepted: January 25, 2016; Published: February 12, 2016

*Corresponding author: Clare S. Allely, Centre for Health Sciences Research, University of Salford, Salford, England M6 6PU; and affiliate member of the Gillberg Neuropsychiatry Centre, Sahlgrenska Academy, University of Gothenburg, Gothenburg, Sweden. Tel: +44 $1612950112 ;$ E-mail: c.s.allely@salford.ac.uk

\begin{abstract}
Within correctional/confinement settings, screening in order to detect Fetal Alcohol Spectrum Disorders (FASD) or Alcohol-Related Neuro Developmental Disorder (ARND) is frequently lacking. The courts may not recognize FAS/ ARND which leads to inappropriate sentences for this vulnerable group. The primary objective of this systematic PRISMA review was to explore the peer reviewed literature which has investigated FASD in the criminal justice system. The present systematic PRISMA review explores the peer reviewed literature published since 1997 which has investigated using four databases in addition to separate searches conducted on 'Google Scholar' using specific search criteria. Only five studies were identified in this review which investigated the prevalence of FASD in an offender population. Only two studies were identified which investigated the psycho-legal abilities of offenders with FASD. Only three studies were identified which examined prenatal exposure to alcohol as potential risk factor for adverse outcomes. Only one study was identified which investigated the knowledge and attitudes of FASD in criminal justice professionals. Overall, the studies identified in this review highlight the urgent need for the development of screening tool(s) for FASD which have demonstrated validity and for the implementation of routine screening for FASD. In terms of the implication for further research and practice, the studies identified in this review highlight that training needs in relation to FASD in correction systems are substantial and require immediate attention. Important issues in formulating and implementing treatment programs for people with FASD in the corrections systems are also discussed.
\end{abstract}

Keywords: Fetal Alcohol Syndrome; Criminal justice system; FASD; FAS; Judges; Prosecutors; Court; Sentencing

\section{Introduction}

Approximately 5-10\% of individuals with significant prenatal alcohol exposure will have Fetal Alcohol Spectrum Disorder (FASD). FASD is not a diagnosis but is a term which is used to refer to a number of diagnostic categories including: Fetal Alcohol Syndrome (FAS), Partial Fetal Alcohol Syndrome (pFAS), AlcoholRelated Neuro Developmental Disorder (ARND) and AlcoholRelated Birth Defects (ARBD) [1]. Individuals with FASD often experience a number of childhood adversities such as neglect and abandonment and often become involved in the criminal justice system [2]. The diagnostic criteria for FASD include: (a) three specific facial abnormalities (e.g., short palpebral fissures or eye openings, flat philtrum or groove below the nose, and small upper lip circumference); (b) prenatal or postnatal growth deficit (i.e., significant deficit in height and/ or weight); and (c) Central Nervous System (CNS) damage. Structural, neurological, or functional assessments provide three ways in which CNS damage is assessed [3].

It has been estimated that individuals affected by FASD are between 19 and 40 times more likely to become involved in the Criminal Justice System [4]. This can create a number of unique challenges and difficulties, for criminal justice professionals, given the nature of the disorder [5]. Lack of adequate executive functioning e.g. understanding the consequences of inappropriate behaviors, and poor impulse control are often the primary reasons that bring individuals with FASD in contact with the Criminal Justice System [6,7]. Such behavioral problems can present as unintentional rule breaking, confabulation and inappropriate sexual behavior. The latter becomes particularly evident during adolescence as the gap between cognitive and chronological age in these subjects increases [2]. Furthermore, lack of understanding and appreciation of legal rights poses additional challenges to individuals with FASD. Fetal alcohol spectrum disorders are shown to cause poor linguistic and communication abilities, both from the expressive standpoint and the receptive one. In other words, individuals affected by FASD experience difficulties understanding and forming coherent communication patterns [8]. Consequently, such individuals may be more easily coerced into improper interrogation procedures 
which can ultimately result in improperly obtained confessions, on the part of the police, due to the suggestibility of FASD affected individuals [9]. FASD also affects an area of experiential learning defined as adaptive functioning. This concept refers to the ability to learn to adapt behavior based on previous experiences. Impaired adaptive functioning also causes these individuals to experience difficulties with self-regulation and impulse control in an unstructured environment e.g. their wider social environment. It is, in fact, very common for an individual with FASD to re-offend and be arrested on multiple occasions due to the inability to make proper associations between actions and their resulting outcomes $[10,11]$. Another important aspect of FASD, of relevance to the Criminal Justice System, is the subject of confabulation. As defined in [2], confabulation is the act of honestly lying or providing information based on inaccurate memories. Confabulation in individuals affected by FASD often results in incorrect testimonies and wrongful conviction and incarceration [2]. Thus, the Criminal Justice System must play a very important role; deficits associated with FASD, that are often the cause of criminal conduct, must be considered to ensure appropriate sentencing on the part of Criminal Justice professionals [11]. However, findings by [12] highlight the lack of knowledge and awareness on the part of professionals, working within the Criminal Justice System, about FASD and the behaviors associated to it. As a consequence, individuals with FASD tend to be over represented in the criminal population [13].

Although mental ill health is widely acknowledged to be a problem in modern society, research findings in this area indicate that the Criminal Justice System is presented with a number of challenges with regard to the identification of mental health disorders. Offenders, in fact, have been shown to have higher rates of mental health disorders than the general community and that such disorders are often not properly identified during the criminal justice processes [14]. Furthermore, findings have highlighted that prevalence of mental ill health is even higher in offenders remanded prior to trial [14-16]. Research has also been commissioned in order to assess the level of screening and the instruments used across jurisdictions by criminal justice agencies. The results suggest that, although assessments occur in all jurisdictions and sectors, there is very little consistency with regard to the manner in which offenders are assessed [14]. Studies over the last two decades have shown that individuals with FASD have Neuro developmental impairments that increase the risk of criminal and antisocial behaviors. As a consequence, it is generally accepted that these individuals are likely to represent an increasingly sizable minority within the criminal Justice System [16]. In many cases, identification of FASD can present a number of challenges due to the lack of the characteristic facial features e.g. small eye openings, a thin upper lip and flat philtrum (the area between the upper lip and the nose). Such features, in fact, are normally only associated with the most severe form of Prenatal Alcohol Exposure (PAE) known as Fetal Alcohol Syndrome or FAS [2]. In addition, it is not uncommon for these features to lose their distinctiveness and attenuate, even in cases of full FAS, as the individual grows older [2]. Research shows that Alcohol-Related Neuro Developmental Disorder (ARND), a subset of FASD, is the most common outcome of prenatal exposure to alcohol [16]. In this population, secondary disabilities, that is to say behavioral manifestations of the Neuro developmental disorder, are often mistaken for intentional criminal and antisocial conduct. These individuals may present as showing no remorse and are unengaged with regard to interrogations and criminal justice proceedings [16]. Individuals with ARND, in fact, do not show any of the physical characteristics that could allow an easier recognition of a disorder associated with prenatal alcohol exposure [5]. Further diagnostic and identification difficulties are associated to the challenges relative to obtaining an accurate history. Current diagnostic criteria preclude the possibility of a diagnosis of ARND if evidence of maternal alcohol abuse during pregnancy is not provided [11].

If FASD is not identified, behaviors such as showing no interest in severity of charges and showing incongruence between affect and gravity of the situation will cause a defendant to present poorly to the judge, prosecutor or probation officer. This, in turn, may result in the defendant presenting as having no mitigating circumstances during sentencing [17]. Despite the fact that a diagnosis of FASD and the resulting cognitive deficits are likely to have been directly relevant to offence conduct and to post-arrest competency, screening for Prenatal Alcohol Exposure (PAE) by criminal justice agencies remains relatively rare [17]. Adult diagnostic and assessment services for FASD remain relatively few worldwide despite four decades of relevant work in this area. There seems to be a general lack of common assessment frameworks specific to adults, which are culturally appropriate and applicable to a more widely diverse population in a range of different settings [18].

Within correctional settings, screening in order to detect FASD is frequently lacking $[6,19,20]$. Studies indicate that many professionals working in the criminal justice system do not have sufficient understanding and training relating to FASD [15,2123]. Epidemiological studies estimate the prevalence of FASD in the United States to be between $1 \%$ and $5 \%$ of the population [24]. Overall, it has been highlighted that there is currently an 'invisible problem plaguing the criminal justice system' [2]. Despite an increasing recognition of FASD within the criminal justice system, it is still concerning that in a review of cases on the Legal Issues Resource Center website (http://depts.washington. edu/fadu/legalissues)maintained by the Fetal Alcohol and Drug Unit at the University of Washington only just over 100 cases in the United States over the past two decades have raised FASD as a defense issue indicating that many individuals go undetected in the criminal justice process [3]. This systematic PRISMA review will explore studies which have investigated FASDs in the criminal justice system. To the authors knowledge, this is the first systematic review to investigate specifically studies which have adopted an experimental design (for instance, used an incarcerated sample to investigate the prevalence of FASD) to investigating FASD in relation to some aspect of the criminal justice system.

\section{Methods}

Internet-based bibliographic databases (Interface-Ebsco 
host Research Databases: MEDLINE; PsycARTICLES; Psychology and Behavioral Sciences Collection and PsycINFO) were searched in order to access studies which investigated FASD in the criminal justice system. The flowchart below outlines the process of eliminating non-relevant papers following PRISMA guidelines see Figure 1 below. The development of the PRISMA (Preferred Reporting Items for Systematic reviews and MetaAnalyses) guidelines was carried out by an international group which comprised of experienced authors and methodologists. The PRISMA Statement consists of a 27 -item checklist and a four-phase flow diagram. The checklist consists of items which are considered important to ensure transparent reporting of a systematic review.

The search included all publications published between 1997 and 2015. Duplicates were excluded prior to the retrieval of references. Searches on all five databases were originally conducted on the $22^{\text {nd }}$ September 2015. The following search criteria were entered into the five databases:

Title (TI) "Fetal Alcohol Spectrum Disorder*" OR FASD OR FAS OR "Fetal Alcohol Syndrome*” OR FAE OR "Fetal Alcohol Effect*" OR "Alcohol-Related Neuro Developmental Disorder" OR ARND OR "Alcohol-Related Birth Defects" OR ARBD OR "Fetal Alcohol Spectrum Disorder*" OR "Fetal Alcohol Syndrome*" OR "Fetal Alcohol Effect*" AND Title (TI) "criminal justice system" OR offend* OR crim* OR prison*. The search returned: 21 . After duplicates were removed there were 14 Fetal is used here as it is the American spelling of the word fetal.

In addition to these database searches, numerous permutations of FASD and other search terms relating to offender populations and the criminal justice system were entered into Google Scholar and thoroughly searched for articles which were not identified through the database searches, for instance, "fetal alcohol syndrome" AND "criminal justice system"; "fetal alcohol syndrome" AND offending, FAS AND "offending", FAS AND "criminal justice system", etc. The reference section was carefully examined for potential relevant studies in each relevant systematic review, literature review or commentary paper. A number of references contained in the papers identified as relevant from the database searches were also examined for possible inclusion in this review. Both authors (CSA and PG) screened the papers for possible inclusion in the review. Given the relatively little research in this area it was decided that this review would be more inclusion than exclusion.

\section{Abstracts for Each Reference were Obtained and Screened using the Following Criteria}

\subsection{Inclusion criteria}

1. Human study population

2. Investigated FASDs in the criminal justice system

\subsection{Exclusion criteria}

1. Paper not published in English

2. Dissertations

3. Book reviews
4. Review papers (however, as mentioned above, review papers were screened, including reference section, for articles which meet the inclusion criteria for this review).

\section{Screening}

In the first stage, papers were rejected which:

- Did not include an investigation of FASD (FAS, ARND, etc) in offender populations or relation to some aspect of the criminal justice system (e.g., judges understanding of FASD in the court process).

- Were book reviews, books, dissertations, commentary papers or review papers.

- $\quad$ For the next stage papers were going to be rejected which:

- Were commentary papers or reviews (not clear from reading the abstract alone).

Lastly, review papers and book chapters which were clearly reviews were excluded and if they were relevant they are covered in the introduction and discussion section. In particular, those reviews or commentary papers which outlined some clinical recommendations are covered in the discussion section under the section clinical recommendations. They were not experimental studies specifically investigating FASD in relation to the criminal justice system so were not included in the result sections of this review which is focused on studies which have been conducted within the field of FASD in the criminal justice system. Full documents were obtained for the remaining records.

\section{Results}

A modest number of studies were identified in this review which investigated some aspect of FASD in relation to some component of the criminal justice system $(n=11)$. A number of studies were reviews and so did not meet the inclusion criteria for discussion in the results of this review. However, all reviews were examined for any key information such as recommendations for legal and mental health professionals working with an offender or defendant with suspected or diagnosed FASD.

\section{Studies investigating the prevalence of FASD in offender populations}

In corrections systems, obtaining accurate prevalence estimates of FASD is a challenge [25,26]. Only five studies were identified in this review which investigated the prevalence of FASD in an offender population [13,26-29]. Fast, et al. [26] investigated the prevalence of FAS/ FAE (FAE, Fetal Alcohol Effect) among youths who were remanded to a forensic psychiatric inpatient assessment unit over one year. The sample comprised 287 youths, between the ages of 12 and 18 years. Each youth underwent a physical examination that involved measuring weight, height and head circumference. The Wechsler intelligence scale (either the Wechsler Intelligence Scale for Children, Third Edition or Wechsler Adult Intelligence Scale, Revised, [30]) was administered as part of the psychological assessment. Diagnosis of FAS was based on the criteria of Sokol 


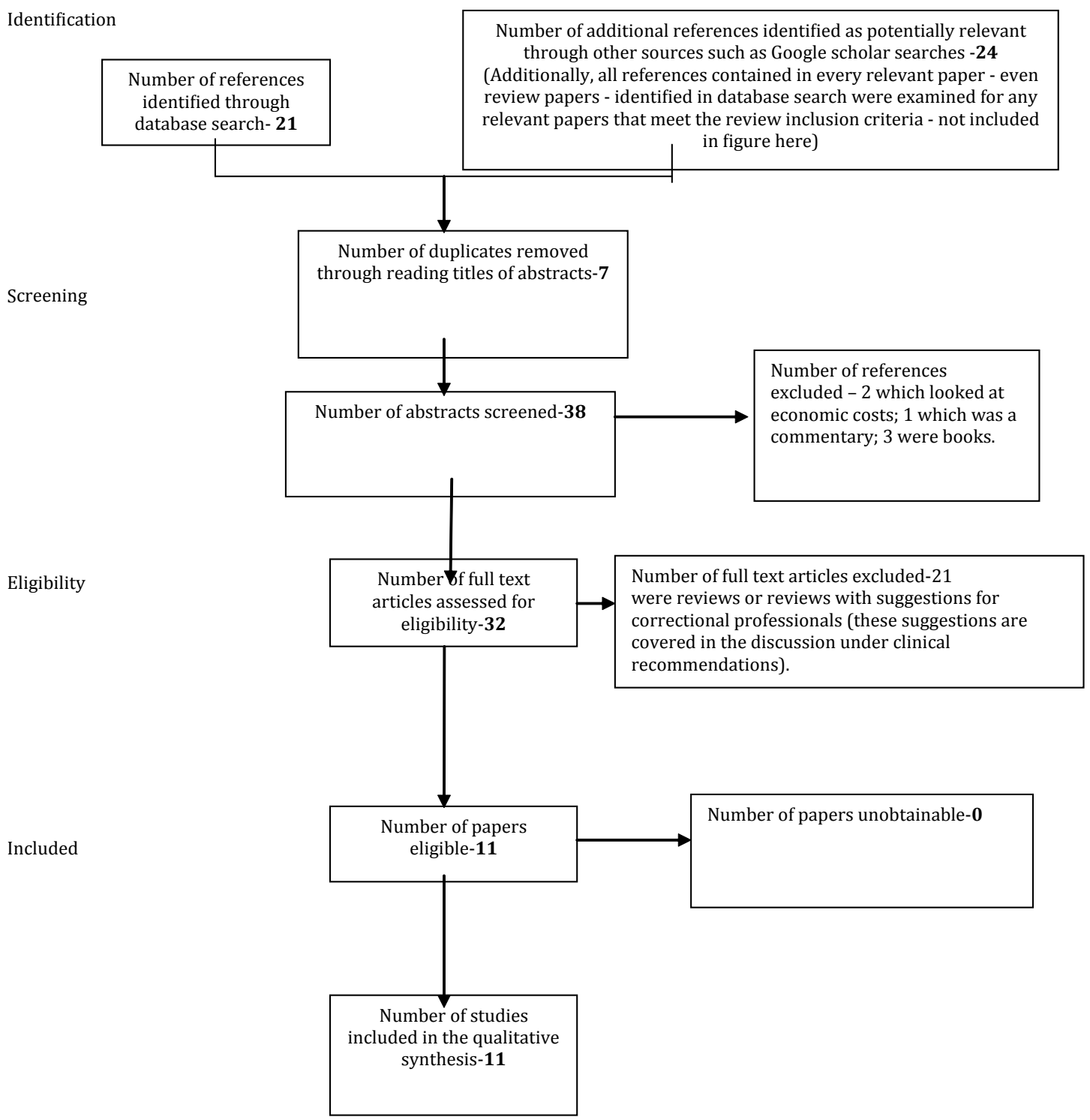

Figure 1: Flow of Information through Systematic Review.

and Clarren, [31]. Youths who had significant prenatal alcohol exposure but did not present all the characteristics for full FAS, received a diagnosis of FAE using Clarren and Smith, [32] diagnostic guidelines. However, some clinicians and researchers have argued that these diagnostic criteria and guidelines are not sufficiently specific in order to be certain of diagnostic accuracy and precision. Instead, they represent an approach to diagnosis which is more gestalt in nature (e.g., [33]). The results indicated that of the 287 youths, 67 (23.3\%) had an alcohol-related diagnosis. Specifically, three $(1.0 \%)$ had received a diagnosis of FAS and $64(22.3 \%)$ had a diagnosis of FAE. Findings by Fast and colleagues, [34] demonstrate the over-representation of young offenders with FASDs in the criminal justice system.

Three programs conducted in the United States which screen for FASD in juvenile offenders and then diagnosed and implemented appropriate interventions are discussed by Bisgard, et al. [27]. The three programs administered an adaptation of the screening tool which was developed by an Expert Panel on FASD Screening in Juvenile Courts. The first program to be covered here is the one carried out by The Juvenile Delinquency Court of the Seventeenth Judicial District of Colorado which screened 718 delinquent youth for FASD in August 2010. Positive screening results for prenatal alcohol exposure was found in 183 (25\%) of the total screened. Seventy-nine (43\%) completed the full FASD diagnostic evaluation. Forty of these $(50 \%)$ received a diagnosis of FASD and they received services and planning following the recommendations from the diagnostic evaluation for FASD. Importantly, none of the clients refused interventions following a diagnosis. In the second program, conducted at the juvenile justice systems in Hennepin County, Minnesota, screening for prenatal 
alcohol exposure in the Juvenile Justice system from August 2008 through September 2010, 148 adjudicated youths were screened for Fetal Alcohol Spectrum Disorder (FASD). Out of the 148, 60 $(41 \%)$ were screened positive for prenatal alcohol exposure. Full FASD diagnostic evaluations were completed on 48 (80\%) youths. Forty-six (96\%) received a diagnosis of FASD. Thirty-five $(76 \%)$ of the youths received services and planning following recommendations from the FASD diagnostic evaluation. A total of 11 clients (24\%) refused interventions following their diagnosis. The third program involved three organizations in Lucas County, Ohio. Initial screening indicated that the estimated generalized total incidence of FASD was 51 (66\%) out of 77 youth placed at a Youth Training Center (YTC) in the 24 month period and for 42 of those youth who remained residents at the YTC 71\% received positive screening results for FASD [21]. Overall, these findings highlight that the raising of awareness and the identification of youths with FASD who become involved in the juvenile courts is crucial [27].

Burd and colleagues, [12] conducted a study which involved each province's or territory's corrections system in Canada to complete a questionnaire on the demographics of the population and services in relation to FAS. Eleven of the thirteen provinces or territories invited to participate took part which resulted in a total population of offenders of 148,797. Based on the total population, a reported diagnosis of FAS was identified in only 13 inmates who indicate a prevalence rate of 0.087 per 1,000 populations. The correction system in the Yukon Territory estimated that $2.6 \%$ of offenders had FAS [13]. In their comprehensive study in the United States, Burd and colleagues, [28] had each state and four major cities' corrections systems complete a questionnaire which explored a variety of areas including: the prevalence of FAS and ARND in the offender population, availability of screening and diagnostic services to detect offenders with FAS and staff training needs in relation to FAS. In the 54 entities, the total population comprised of 3,080,904 inmates. Burd and colleagues, [28] estimate the potential prevalence of cases of undiagnosed FAS and partial FAS (pFAS) in the United States corrections system population may range from somewhere between 1,540 and 28,036 [28]. These low estimates of the prevalence of undiagnosed FAS and pFAS compared to the much higher rate identified through deliberate screening of inmates emphasis the invisible 'hidden nature' of the FASD. Burd and colleagues, [28] survey of services for FAS and ARND in the corrections system indicates that identification of expected cases of FAS or ARND is lower than 1\%. The reported staff training needs were substantial. This survey draws attention to the "high unmet needs to screen, identify, and treat offenders with FAS and ARND" in correction systems. Burd and colleagues, [28] further stress the invisibility of this disorder but highlight that the facial features which are characteristic of FAS are frequently not exhibited in many individuals with a diagnosis of FASD [28].

Lastly, Rojas and Gretton, [29] investigated the background, offence characteristics, and criminal outcomes of Aboriginal $(n=102)$ and non-Aboriginal $(n=257)$ youths (aged between 12 and 18 years) who engaged in sexual offending behavior and were ordered to attend the Youth Sexual Offence Treatment
Programme (YSOTP) located either in Burnaby or Prince George, in British Columbia between 1985 and 2004. Aboriginal youths were nearly seven times more likely than non-Aboriginal youths to exhibit evidence of FASD (27\% versus 4\%, respectively) [29].

\section{Prenatal exposure to alcohol: A risk factor for antisocial behavior?}

Only two studies were identified which examined prenatal exposure to alcohol as potential risk factor for adverse outcomes [35-37]. Momino and colleagues, [36] found prenatal exposure to alcohol to be only one of the risk factors for antisocial behaviour. Other social factors (e.g., family and domestic violence) also associated with maternal drinking, were highly frequent in the risk group. The sample consisted of 262 institutionalized male Brazilian adolescents and 154 male students between the ages of 13 and 21 years. Measures included a questionnaire, specific to alcohol consumption during pregnancy and family alcohol history, Raven's Standard Progressive Matrices, used as intelligence measurement tool, and standard physical measurements (e.g., weight, height and head circumference). The diagnostic guidelines of the Institute of Medicine were adopted for the diagnosis of FAS [38]. Findings showed no statistically significant difference in maternal drinking between the institutionalized adolescent male group and the male student group. Alcohol consumption during pregnancy was reported by $48.8 \%$ of the mothers for the experimental group (mothers of the institutionalized adolescents) and by $39.9 \%$ of the mothers for the control group (school students). Domestic violence, familial criminality and child abuse (all $<0.001$ ) were the only pregnancy quality and familial history factors found to be significantly different between the adolescents with criminal behavior (FASE) group and the school students (controls) group. In this study, no individuals were identified with a clear FAS diagnosis. However, in the institutionalized adolescent, signs indicative of FASD were more frequent. However, there needs to be caution with this conclusion. It is unclear from this study whether alcohol exposure in utero is a risk factor for institutionalization in adolescence given that there was no reliable difference in maternal use of alcohol during pregnancy between the institutionalized and non-institutionalized adolescents. Findings suggest that offending behavior is more associated with interactions between environmental and social factors including prenatal alcohol exposure [36].

Streissguth and colleagues, [35] examined a group of 415 individuals (median age 14 years, range 6-51; median IQ 86, range 29-126) with FAS and FAE. Using the Life History Interview with knowledgeable informants, Streissguth and colleagues, [35] investigated five adverse outcomes and 18 related risk/ protective factors. Trouble with the law was found in $67 \%$ of the adolescents and $87 \%$ of the adults. Additionally, $35 \%$ of the adolescents and adults had been in prison. They also found that, compared to an individual with full FAS, brain function may actually be more impaired in an individual with an FASD who fails to meet the diagnostic criteria for the more recognizable full FAS. Findings revealed that $80 \%$ of the 415 individuals were brought up by someone other than their biological mothers. The life 
span prevalence for both adolescents and adults for 'Disrupted School Experiences' was 61\%, 60\% for 'Trouble with the Law, $50 \%$ for 'Confinement' (in detention, jail, prison, or a psychiatric or alcohol/drug inpatient setting), $49 \%$ for multiple instances of 'Inappropriate Sexual Behaviors' and 35\% for 'Alcohol/Drug Problems'. The clinical importance of identifying FAS or FAE as early as possible is emphasised by the findings that receiving the diagnosis of FAS or FAE at an earlier age and by being raised within a supportive and stable environment increases the odds of avoiding these adverse life outcomes by 2-4 fold [35].

Lastly, Corrado and McCuish, [37] investigated the developmental and offending outcome differences between incarcerated Canadian FASD $(n=58)$ and non-FASD $(n=456)$ youth. They found that the incarcerated youth with FASD were more likely to have numerous experiences which are considered to be risk factors for offending. Such criminogenic factors included: placement in foster care, presence of a co morbid behavioral disorder, low levels of self-control, a negative self-identity, and an earlier onset of alcohol use. Interestingly, when other criminogenic factors were accounted for (such as placement in foster care), FASD was not found to be associated with early onset and frequent offending. The key findings was that FASD youth were only at risk of early and frequent offending if they first had experienced other negative outcomes. The researchers emphasize that this is positive in that it highlights that intervention programmers focused on targeting the criminogenic risks factors is the key to prevention of offending in youth with FASD [37].

\section{Evaluating the psycho-legal abilities of young offenders with FASD}

Only two studies were identified which investigated the psycho-legal abilities of offenders with FASDs [8,39]. In a group of young offenders with FASDs, McLachlan, et al. [8] investigated the psycho-legal abilities relevant to interrogation and adjudication in addition to assessing the offender's insight and confidence regarding their psycho-legal abilities. A variety of measures were used including: Miranda Instruments, Fitness Interview Test - Revised FIT-R [40], Wechsler, [41] abbreviated scale of Intelligence (Psychological Corporation, 1999) and the Wide Range Achievement Test 4th Edition WRAT-4 [42]. The sample comprised of 100 young offenders recruited from two Canadian provinces (19 females and 81 males, age range 12 to 23 years, $\mathrm{M}=17.53$ years, $\mathrm{SD}=1.59$ ). 50 young offenders with a FASD diagnosis were compared with a control group of 50 young offenders not suspected of having experienced PAE. Participants were included in the FASD group if they had been diagnosed by an interdisciplinary team which followed the Canadian Diagnostic Guidelines for FASD [43], and they were currently or recently (within the last three years) involved in the criminal justice system. Inclusion criteria for the comparison group were individuals not suspected of PAE and also not currently or recently (within three years) involved in the justice system. Results indicated that most participants in the FASD group demonstrated significant impairments in understanding and appreciation of their arrest rights. $84.0 \%$ of the young offenders in the FASD group failed at least one item within each of Grisso's understanding instruments as opposed to $64.0 \%$ in the control group. Results further indicated that no statistically significant difference was found between participants with FASD ratings of both understanding and waiver decisions compared to the control group's understanding and waiver decisions. The term waiver is adopted across a variety of legal contexts. A waiver refers to a unilateral act of one individual which results in the surrender of a legal right. There was a positive correlation (although there was no significant difference) between selfassessed understanding and total scores on Grisso's instruments in both the FASD group and the control group. However, there was a significant difference found in the ability to make informed waiver decisions between the control group and the FASD group. In order to assess predictors of rights comprehension, a number of correlations were conducted between IQ, reading ability, legal experience, and scores on Grisso's four instruments. Significantly lower scores across all four instruments were found in the participants with FASD compared to the comparison group. This supports the theory that offenders with FASD may be impaired in their ability to both understand and appreciate their rights. In addition, $76.0 \%$ of the FASD group demonstrated impaired performance on the understanding scale of the FIT-R. Scores from the appreciation and communication scales were also impaired in $24.0 \%$ of the FASD group. Overall, $76 \%$ of the FASD group demonstrated impaired performance on at least one of the components of the FIT-R compared with only $28.0 \%$ of the control group [8].

Another study involved a small pilot study conducted in the United States which investigated the role of suggestibility as a psychological vulnerability in defendants with FASD who were involved in a pre-trial or post-conviction adjudication process. Brown, et al. [39] pilot study consisted of seven adults who were referred for multidisciplinary assessment by experts on FASD by counsel. All seven participants were male and ages ranged from 17 to 53 years of age, average age 29 years (African American, $\mathrm{n}=4$ and Caucasian, $\mathrm{n}=3$ ). Following the multidisciplinary assessment, only one of the seven participants received a diagnosis of full FAS. The remaining six participants received a diagnosis of Partial FAS. Wide variation in intellectual functioning (measured using full scale IQS) was found in the group (ranging from 61 to 112 , mean $=83$, SD = 17). The Gudjonsson Suggestibility Scale 2 , GSS2 was used, rather than the GSS1, as it is commonly used with individuals with intellectual impairments. Findings indicate that in interrogative situations/ contexts, individuals with FASD may be highly suggestible [39]. While an important contribution to the relatively small field, it is necessary when interpreting the findings from this study that the significant methodological limitations of the study (e.g., small sample size and the lack of comparison group) is taken into consideration.

\section{Knowledge and attitudes of FASD in criminal justice professionals}

Only one study was identified which investigated the knowledge and attitudes of FASD in criminal justice professionals [44]. To assess the knowledge and attitudes (including training/ 
educational needs) of FASD in criminal justice professionals, Cox, et al. [44] gathered information from New Brunswick Criminal Justice System professionals, in particular Provincial Judges and Prosecutors between June 2005 and June 2006. Nineteen individuals out of a total 53 Prosecutors agreed to take part. Forty-six percent of the total population of all of the Judges in the New Brunswick Provincial Court and all of the Crown Prosecutors in New Brunswick Public Prosecutions took part. Twelve percent of Judges and none of the surveyed Prosecutors were aware of the 2005 Canadian Guidelines for FASD Diagnosis. Less than $50 \%$ had ever heard of the term FAE. Only $6 \%$ of Judges and none of the Prosecutors reported being familiar with the term ARND or Alcohol Related Neuro developmental Disorder. The physical characteristics of FASD were not known in nearly $50 \%$ of respondents. In terms of feeling "prepared" to handle cases involving someone with FASD (suspected or diagnosed, etc), $40 \%$ of the Judges and only $26 \%$ of the Prosecutors reported feeling "prepared". Merging the Prosecutors and the Judges to create one sample, it was found that the Prosecutors and the judges to a greater degree highlighted the importance of some supports such as more research information (68\%). Thirty-seven percent of Prosecutors wanted more diagnostic information to be available and $42 \%$ reported that the development of practice guidelines would be important. Only three judges and three Prosecutors reported knowing where to refer an individual for FASD diagnosis. Moreover, knowing where to refer an individual with FASD for treatment was only reported by one of the judges and prosecutors. Nearly all the justice professionals were in agreement that such treatment options would be useful. Judges and Prosecutors, who reported being informed about Correction Services Canada's report regarding the prevalence of FASD within the criminal justice system, were asked, based on their own professional experience, the prevalence of FASD related disabilities. Interestingly, the Judges and Prosecutors responses varied widely from as little as a few to as high as $40 \%$, with the plurality category being "between $10 \%$ and $19 \%$ ". The need for a more coordinated approach among justice and health professionals and for sessions on FASD disabilities with other legal professionals to develop a New Brunswick "approach" was unanimously considered to be valuable. At least $80 \%$ suggested that a useful initiative would be the implementation of a Mental Health court [44].

\section{Service availability for offenders with FASD}

Two studies were identified which examined the service availability for offenders with FASD in the criminal justice system and both were from the same research group $[13,28]$. The study by Burd and colleagues, [12] is also included in the prevalence section. Availability of services for diagnosis and management of individuals with FAS, ARND or FAE in the Canadian corrections system is relatively under researched. In order to investigate this, Burd and colleagues, [12] conducted a study which involved each province's or territory's corrections system to complete a questionnaire on the demographics of the population and services in relation to FAS. Eleven of the thirteen provinces or territories invited to participate took part which resulted in a total population of offenders of 148,797. The mean rate of substance abuse was found to be $50.5 \%$ based on the 11 entities which responded. Based on the total population, a reported diagnosis of FAS was identified in only 13 inmates who indicate a prevalence rate of 0.087 per 1,000 population. The correction system in the Yukon Territory estimated that $2.6 \%$ of offenders had FAS [13]. A screening program for FAS in the corrections system was not reported by any of the participating entities. Access to diagnostic services for FAS was only reported in three of the eleven entities (27.3\%). This study emphasized the substantial level of staff training needs required [13]. The study by Burd and colleagues, [12] found that specialized programs for pregnant women with substance abuse problems were reported by two (15.4\%) corrections systems and three $(23.1 \%)$ community corrections systems. Additionally, Burd and colleagues, [28] in their sample comprising of 3,080,904 inmates (study described earlier in prevalence section) found substance abuse in $60.1 \%$ of the offenders. In the corrections facilities, $46.3 \%$ reported having programs for pregnant women. In the community facilities, $29.6 \%$ reported having programs for pregnant women. Crucially, a screening program for FAS in the corrections system was only reported by one program (1.9\%). Access to diagnostic services for FAS in the corrections facilities was only reported in four programs (7.4\%). A diagnosis of FAS was only reported in one offender out of a total of $3,080,904$ offenders.

\section{Discussion}

Overall, only eleven studies were identified which investigated FASDs in relation to some aspect of the criminal justice system. Specifically, five studies which investigated the prevalence of FASD in an offender population [13,26-29] two which investigated the psycho-legal abilities of offenders with FASDs [8,39]; two which examined prenatal exposure to alcohol as potential risk factor for adverse outcomes (Streissguth, et al. [35]; Momino, et al. [36]; Corrado, et al. [37]; one which investigated the knowledge and attitudes of FASD in criminal justice professionals Cox, et al. [44] and lastly, two studies which examined the service availability for offenders with FASD in the criminal justice system and both were from the same research group (Burd, et al. [13,28]. Overall, this review highlights the paucity of research that has been conducted across numerous aspects of the criminal justice process in relation to this vulnerable, largely "invisible" group.

Studies identified in this review indicate that FASD is not being identified in individuals involved in the criminal justice system. The findings of only one offender reported as having a diagnosis of FAS from a total surveyed 3.08 million offenders in the United States really highlights this [28]. A number of areas were found to be lacking, including: infrastructure capacity, staff awareness and access to screening and diagnostic services. Systematic screening for FASDs is required in correction systems $[13,28]$. Additionally, the survey conducted by Cox and colleagues, [44], which aimed at determining Judge's and Prosecutor's knowledge, attitudes behaviors and training needs relative to cases of FASDs informed a number of recommendations including the need for the development of a coordinated approach to FASD in the Criminal Justice System and the development of clear practice guidelines. They also recommend improving the 'professional preparedness' 
to care for individuals with FASD, the training of Judges and Prosecutors about FASD, when and where to refer for diagnosis and the long-term secondary disabilities associated with FASD and the development of a Wellness or Mental Health Court to work with individuals with FASD in Criminal Justice System [44]. Furthermore, McLachlan and colleagues, [8] findings support the view that the psycho-legal abilities in individuals with FASD needs to be evaluated using an individualized and comprehensive forensic assessment.

\section{Sentencing offenders with fetal alcohol syndrome disorder}

The courts may not recognize FAS/ ARND which leads to inappropriate sentences for this vulnerable group. While there are some courts which recognize FASD as being one of a number of mitigating and aggravating factors, others do not consider that individuals with FAS or ARND should receive any special consideration. There is, however, significant variation in the way that courts who do acknowledge the presence of FAS/ ARND actually take this into consideration. There are also multiple challenges and barriers faced by these courts due to the poor level of cooperation and resources in place within the correctional system in order to support and provide appropriate treatment for offenders with FAS/ ARND [45]. Individuals with FASD can be misunderstood in court, are at increased risk of experiencing victimization in prison and can often be mismanaged in the transition back to the community - all of which can be minimized if the professionals working with the individual with FASD have received education and training regarding the nature and implications of FASD [46].

The development of a comprehensive medical-legal report can aid the judge and other criminal justice professions involved with an individual with FASD. Fast and Conry, [46] argue that for individuals with FASD, going to court can actually be counterproductive in that the duration between the time the offence was committed and sentencing is so extracted that individuals with FASD may be unable to fully grasp that the two are connected and therefore fail to recognize the consequences of their offence. This is also the case for offences committed prior to the trial or sentencing Fast and Conry, [46]. Mela and Luther, [47] propose that, in order to improve on current practice, a diminished responsibility defense and verdict which takes into consideration the 'grey zone' between 'knowing' and 'not knowing' based on Neuro cognitive disparities in FASD is crucial [47]. Lastly, the study identified in this review by Cox and colleagues, [44] emphasizes that Judges and Prosecutors require more education and training in order to assist them in their work with individuals with FASD who become involved in the criminal justice system. Another area which was considered to be beneficial by Judges and Prosecutors was access to accurate and timely assessment and diagnoses of FASD [44].

\section{Clinical recommendations}

A number of studies found in this review (but not reviewed in the results section as they were review paper) recommend that all correctional and custodial staff are trained to identify inmates who may have FASD (e.g., Fast \& Conry, [46]; Brown, et al. [5] and also recognize how it can impact on the offender's ability to understand and adhere to rules and probation orders [53]. Burd and colleagues, [21] highlight in their paper that the reported training needs in correction systems are substantial, requiring immediate attention Burd, et al. [21].

FASD Experts recommend the use of an informal checklist which includes empirically validated factors found to be associated with FASD Brown, etal. [3]. The FASD experts screening questionnaire covers five areas including: Offence Conduct (e.g., Impulsive and illogical actions with high risk of detection and Poor exit strategy); Arrest Conduct (e.g., immediately or easily waives rights and over-confesses (suggestible)); Interview with Client (e.g., socially inept, immature, and naïve and Doesn't seem to remember what you tell him/her from appointment to appointment); Prior Legal History (e.g., easily led by more sophisticated peers and illogical offences (e.g., stealing something with little value)); and lastly, Life History (e.g., involvement with child welfare, Adoption/foster or relative placement/juvenile commitment [3].

Moreover, Burd and colleagues, [21] outline important issues to consider when formulating and implementing treatment programs for individuals with FASD in the corrections system (See Table 1.). When working with an individual with a FASD, Brown and colleagues, [17] outline in their paper some communication suggestions which may assist correctional officers. For instance, it is strongly advised to avoid the use of questions requiring a strict "yes" or "no" when attempting to determine comprehension as such questions can hide an individual's capacity to understand what is being asked of them. In an effort to please others, individuals with an FASD will often respond with what they think others want to hear even at their own expense. Therefore it is recommended that correction officers utilize questions which are open ended and also encourage inmates to repeat what they have heard [17].

\section{Psychiatric co-morbidity in young offenders with FASD}

Another important clinical consideration is the degree of co-morbidity in individuals with FASD in the criminal justice system. Conry, Fast and Loock, [34] examined young people in the criminal justice system who had been referred for assessment to a forensic psychiatric facility and found that, for both the controls and the youths with FASD, the most frequently diagnosed psychiatric disorders included: conduct disorder, Attention Deficit Hyperactivity Disorder (ADHD) and substance use disorder. Importantly, all the youths with an FASD also had at least one psychiatric disorder. This study was not included in the review because it was not a peer reviewed article and also could not be obtained by the authors.

\section{Limitations}

It is possible that the search terms utilized in the search for the present review failed to capture all of the relevant research in the field of FASD in relation to the criminal justice system. In order to minimize the risk of missing any relevant articles 


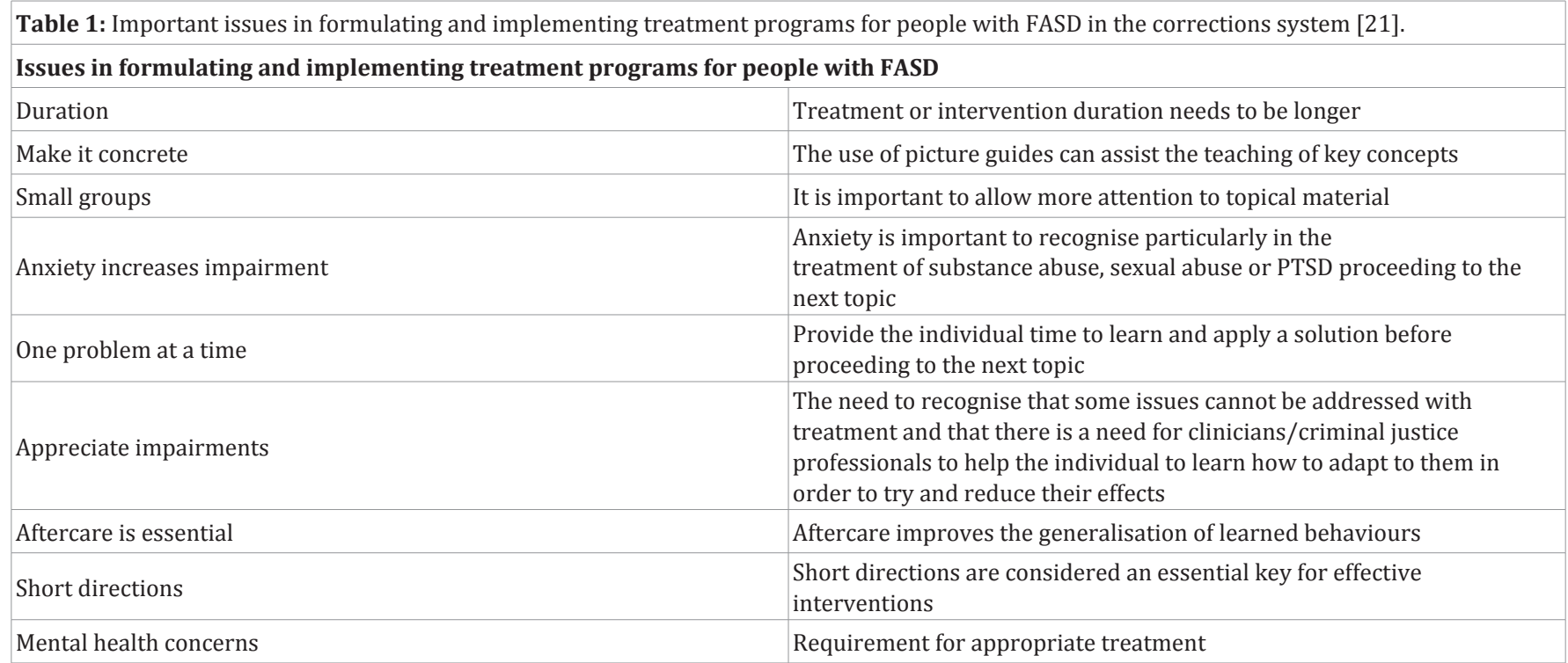

additional searches on 'Google scholar' using specific search criteria as discussed in the methods section were also carried out.

\section{Future Directions}

Fast and Conry, [48] highlight the urgent need for the development of screening tool(s) for FASD which have demonstrated validity. For probation officers, The Asante Center in British Columbia developed a screening and referral guide [49]. Goh and colleagues, [50] evaluated screening tools for FASD, and selected the Asante Centre for Fetal Alcohol Syndrome Probation Officer Screening \& Referral Form as a youth justice screening tool (http://www.asantecentre.org/_Library/docs/Youth Probation_Officers_Guide_to_FASD_Screening_and_Referral_ Printer-Friendly_Format_.pdf). However future studies need to assess this screening tool in terms of its [51]. Lastly, there has also been a paucity of studies investigating the rate of recidivism in offenders with FASD which merit further study [28].

This review covers a relatively modest number of studies which highlight that FASD and offending behavior needs more attention. It also highlights the need for the development of early intervention for youths with FASD in order to mitigate against the risk of offending behavior. Some work on developing programs for youths with FASD is underway. For instance, Soh and colleagues, [52] recently conducted a study which examined the structural brain changes associated with the Alert Program for Self-Regulation in children with FASD. Soh and colleagues, [52] study suggested that Alert is effective in increasing cortical gray matter in children with FASD, most notably in those brain regions underlying response inhibition, outcome monitoring and emotion regulation. This is the first study to identify neuroanatomical changes after treatment for the core deficit in executive functioning in children with FASD which indicates that functional outcome in children with FASD can be impacted on positively by early intervention [52]. Studies such as this highlight the need for further research to inform the most effective interventions for at-risk youths with FASD.

\section{Conclusions}

The studies identified in this review clearly highlight the importance of screening for FASD within correctional settings $[21,53]$. Early recognition of the impairments experience by individuals with FASDs may help lower the over-representation of this vulnerable group within the criminal justice system [48].

\section{References}

1. Stratton KR, Howe CJ, Battaglia FC. Fetal Alcohol Syndrome: Diagnosis, epidemiology, prevention, and treatment. Washington, DC: National Academy of Sciences; 1996.

2. Brown J. Fetal alcohol spectrum disorders in the criminal justice system: A review. The Journal of Law Enforcement. 2014;3(6).

3. Brown NN, Wartnik AP, Connor PD, Adler RS. A proposed model standard for forensic assessment of Fetal Alcohol Spectrum Disorders. The Journal of Psychiatry and Law. 2010;38(4),383-418.

4. Popova S, Lange S, Burd L, Rehm J. Cost attributable to Fetal Alcohol Spectrum Disorder in the Canadian correctional system. Int J Law Psychiatry. 2015;41:76-81. doi: 10.1016/ j.ijlp.2015.03.010.

5. Jerrod Brown, Jay P. Singh. Fetal Alcohol Spectrum Disorder and RiskNeed-Responsively Theory: An Exploratory Discussion. Behavioral Health. 2015;3(2).

6. Brown NN, Connor PD, Adler RS. Conduct-disordered adolescents with Fetal Alcohol Spectrum Disorder intervention in secure treatment settings. Criminal Justice and Behavior. 2012;39(6):770793. doi: $10.1177 / 0093854812437919$.

7. Verbrugge P. Fetal alcohol spectrum disorder and the youth criminal justice system: A discussion paper. Department of Justice. Canada: Youth Justice Research; 2003.

8. McLachlan K, Roesch R, Viljoen JL, Douglas KS. Evaluating the psycholegal abilities of young offenders with fetal alcohol spectrum disorder. Law Hum Behav. 2014;38(1):10-22. doi: 10.1037/ lhb0000037.

9. McLachlan K. An examination of abilities, risks, and needs of adolescents and young adults with Fetal Alcohol Spectrum Disorder 
(FASD) in the criminal justice system. Burnaby, BC: Simon Fraser University. 2012.

10. William J. Edwards and Stephen Greenspan. Adaptive Behavior Alcohol Spectrum and Fetal Disorders. The Journal of Psychiatry and Law. 2010;38(4):419-447.

11. Brown J, Mitchell M, Wartnik A, Russell A. Fetal Alcohol Spectrum Disorder and the Courts: An Introduction for Legal Professionals Behavioral Health. 2015;3(1).

12. Burd L, Cotsonas-Hassler TM, Martsolf JT, Kerbeshian J. Recognition and management of fetal alcohol syndrome. Neurotoxicol Teratol. 2003;25(6):681-688.

13. Larry Burd, Rachael H. Selfridge, Marilyn G. Klug, Tim Juelson, BA. Fetal Alcohol Syndrome in the Canadian corrections system. Journal of FAS International. 2003;1:e14

14. James RP Ogloff, Michael R Davis, George Rivers and Stuart Ross. The Identification of Mental Disorders in the Criminal Justice System. Trends and Issues in Crime and Criminal Justice. p. 334. 2007.

15. Odgers CL, Burnette ML, Chauhan P, Moretti MM, Reppucci ND. Misdiagnosing the problem: Mental health profiles of incarcerated juveniles. Can Child Adolesc Psychiatr Rev. 2005;14(1):26-29.

16. Brown NN, Burd L, Grant T, Edwards W, Adler R, Streissguth A Prenatal alcohol exposure: An assessment strategy for the legal context. Int J Law Psychiatry. 2015;42-43:144-148. doi: 10.1016/ j.ijlp.2015.08.019.

17. Jerrod Brown, Anthony Wartnik, Mario Hesse, Jeffrey Long-McGie, Tina Andrews, Mary Mahoney Weaver, et al. Fetal Alcohol Spectrum Disorder in Confinement Settings: A Review for Correctional Professionals. The Journal of Law Enforcement. 2015;4(4).

18. Townsend, Clare, Hammill, Kate, White, Paul. Fetal alcohol disorder, disability and the criminal justice system. Indigenous Law Bulletin, 2015;8(17):30-33.

19. Bracken DC. Canada's Aboriginal people, Fetal Alcohol Syndrome \& the criminal justice system. British Journal of Community Justice. 2008;6(3):21-33

20. Graham H. Fetal Alcohol Spectrum Disorder and Inappropriate Sexual Behavior. (Master's thesis). Laurentian University, Sudbury, Ontario, Canada. 2014

21. Burd L, Diane KF, Conry J, Andrew D. Williams. Fetal Alcohol Spectrum Disorder as a marker for increased risk of involvement with correction systems. The Journal of Psychiatry and Law. 2010;38(4):559-583. doi: 10.1177/ 009318531003800408

22. Clarren SK, Lutke J. Building clinical capacity for fetal alcohol spectrum disorder diagnoses in Western and Northern Canada. Can J Clin Pharmacol. 2008;15(2):e223-37.

23. Wedding D, Kohout J, Mengel MB, Ohlemiller M, Ulione M, Cook K, et al. Psychologists' knowledge and attitudes about fetal alcohol syndrome, fetal alcohol spectrum disorders, and alcohol use during pregnancy. Professional Psychology Research and Practice. 2007;38(2):208-213. doi: 10.1037 / 0735-7028.38.2.208.

24. May PA, Gossage JP, Kalberg WO, Robinson LK, Buckley D, Manning M, Hoyme HE. Prevalence and epidemiologic characteristics of FASD from various research methods with an emphasis on recent in-school studies. Dev Disabil Res Rev. 2009;15(3):176-92. doi: 10.1002/ ddrr.68.

25. Conry J, Fast DK. Fetal Alcohol Syndrome and the criminal justice system. 2000
26. Fast DK, Conry J, Loock CA. Youth in the criminal justice system: Identifying FAS and other developmental disabilities. J Dev Behav Pediatr. 1999;20(5):370-372.

27.Eileen B Bisgard, Suzette Fisher, Susan Adubato, Meghan Louis. Screening, diagnosis, and intervention with juvenile offenders. The Journal of Psychiatry and Law. 2010;38(4):475-506. doi: 10.1177/ 009318531003800406

28. Burd L, Selfridge R, Klug M, Bakko S. Fetal Alcohol Syndrome in the United States corrections system. Addiction Biology. 2004;9(2):169176.

29. Rojas EY, Gretton HM. Background, offence characteristics, and criminal outcomes of Aboriginal youth who sexually offend: A closer look at Aboriginal youth intervention needs. Sex Abuse. 2007;19(3):257-283.

30. Wechsler D. WISC-III: Wechsler intelligence scale for children: Manual. Psychological Corporation; 1991.

31. Sokol RJ, Clarren SK. Guidelines for use of terminology describing the impact of prenatal alcohol on the offspring. Alcohol Clin Exp Res. 1989;13(4):597-598.

32. Sterling K. Clarren and David W. Smith. Fetal alcohol syndrome. New England Journal Medicine. 1978;298:1063-1067. DOI: 10.1056/ NEJM197805112981906

33. Astley SJ, Clarren Sk. Diagnosing the full spectrum of fetal alcoholexposed individuals: introducing the 4-digit diagnostic code. Alcohol Alcohol. 2000;35(4):400-410.

34. Fast DK, Conry J, Loock CA. Identifying fetal alcohol syndrome among youth in the criminal justice system. J Dev Behav Pediatr. 1999;20(5):370-372.

35. Streissguth AP, Bookstein FL, Barr HM, Sampson PD, O’Malley K, Young JK. Risk factors for adverse life outcomes in Fetal Alcohol Syndrome and Fetal Alcohol Effects. J Dev Behav Pediatr. 2004;25(4):228-38.

36. Momino W, Félix TM, Abeche AM, Zandoná DI, Scheibler GG, Chambers C. Maternal drinking behavior and Fetal Alcohol Spectrum Disorders in adolescents with criminal behavior in southern Brazil. Genet Mol Biol. 2012;35(4 (suppl)):960-965.

37. Corrado RR, McCuish EC. The development of early onset, chronic, and versatile offending: The role of fetal alcohol spectrum disorder and mediating factors. International Journal of Child and Adolescent Health. 2015;8(2):241-250.

38. Hoyme HE, Burden MJ, Andrew C, Saint-Amour D, Meintjes EM, Molteno $\mathrm{CD}$, et al. The effects of fetal alcohol syndrome on response execution and inhibition: An event-related potential study. Alcohol Clin Exp Res. 2009;33(11):1994-2004. doi: 10.1111/ j.1530-0277.2009.01038

39. Brown NN, Gudjonsson G, Connor P. Suggestibility and fetal alcohol spectrum disorders: I'll tell you anything you want to hear. The Journal of Psychiatry and Law. 2011;39(1):39-71. doi: 10.1177/ 009318531103900103

40. Roesch R, Zapf PA, Eaves D. Fitness Interview Test-Revised: A structured interview for assessing competency to stand trial. Sarasota, FL: Professional Resource Press; 2006.

41. Psychological Corporation. Wechsler Abbreviated Scale of Intelligence. San Antonio, TX: Author; 1999.

42. Wilkinson GS, Robertson GJ. Wide Range Achievement Test 4 Professional Manual. Lutz, FL: Psychological Assessment Resources. 2006 
43. Chudley AE, Conry J, Cook JL, Loock C, Rosales T, LeBlanc NF, et al alcohol spectrum disorder: Canadian guidelines for diagnosis. Canadian Medical Association Journal. 2005;172: S1-S21.

44. Cox LV, Clairmont D, Cox S. Knowledge and attitudes of criminal justice professionals in relation to fetal alcohol spectrum disorder. Can J Clin Pharmacol. 2008;15(2):e306-13.

45. Chartrand LN, Forbes-Chilibeck EM. The sentencing of offenders with fetal alcohol syndrome. Health Law J. 2003;11:35-70.

46. Fast DK, Conry J. The challenge of fetal alcohol syndrome in the criminal legal system. Addict Biol. 2004 ;9(2):161-166.

47. Mela M, Luther G. Fetal alcohol spectrum disorder: Can diminished responsibility diminish criminal behaviour? Int J Law Psychiatry. 2013;36(1):46-54. doi: 10.1016/ j.ijlp.2012.11.007.

48. Fast DK and Conry J. Fetal alcohol spectrum disorders and the criminal justice system. Dev Disabil Res Rev. 2009;15(3):250-257. doi: 10.1002 / ddrr.66.
49. Conry J, Kwadwo O. Youth probation officers' guide to FASD screening and referral. Maple Ridge, BC: The Asante Center for Fetal Alcohol Syndrome. 2010.

50. Goh YI, Chudley AE, Clarren SK, Koren G, Orrbine E, Rosales T, et al. Development of Canadian screening tools for Fetal Alcohol Spectrum Disorder. Can J Clin Pharmacol. 2008;15(2):e344-66.

51. Conry J, Asante KO. Youth probation officer's guide to FASD screening and training. 2010. http://www.asantecentre.org/resources.html.

52. Soh DW, Skocic J, Nash K, Stevens S, Turner GR, Rovet J. Self-regulation therapy increases frontal gray matter in children with fetal alcohol spectrum disorder: evaluation by voxel-based morphometry. Front Hum Neurosci. 2015;4(9):108. doi: 10.3389/ fnhum.2015.00108.

53. Fast DK and Conry J. Fetal alcohol spectrum disorders and the criminal justice system. Dev Disabil Res Rev. 2009;15(3):250-257. doi: $10.1002 /$ ddrr.66. 